\title{
Thin Copolymer-Cased Light-Emitting Display Made with Fluorine-Foped Tin Oxide Substrates
}

\author{
Rudolf Lessmann, Ivo Alexandre Hümmelgen* \\ Group of Organic Optoeletronic Devices, Departamento de Física, \\ Universidade Federal do Paraná, C.P. 19044, 81531-990 Curitiba - PR, Brazil
}

Received: December 15, 2003; Revised: May 30, 2004

\begin{abstract}
Seven-segment displays are used to show numerical quantities in electronic equipment, being present in most of the low-end electronics. In this work we describe a novel organic light emitting display made with poly $(1,10$ - decanedioxy -2 - methoxy - 1,2 - phenylene - 1,2 - ethenylene 3,6-dimethoxy - 1,4 - phenylene - 1,2 - ethenylene - 3- methoxy - 1,4 - phenylene) (OPPVDBC), tris(8-hydroxyquinoline)aluminum salt $\left(\mathrm{Alq}_{3}\right)$ and a hole injection layer (PEDOT:PSS: poly (3,4 ethylenedioxythiophene) : poly(styrenesulfonate)). The general device structure is FTO/ PEDOT:PSS/OPPVDBC/Ca/Al or FTO/PEDOT:PSS/OPPVDBC/Alq/ $/ \mathrm{Ca} / \mathrm{Al}$. The FTO (fluorinedoped tin oxide) thin films are transparent (transmittance $>80 \%$ in the visible region of the spectrum), conductive ( $<15 \Omega /$ o for $200 \mathrm{~nm}$ thick films), and present high chemical stability.
\end{abstract}

Keywords: electroluminescence, copolymer, fluorine-doped tin oxide

\section{Introduction}

Since the development of the first organic light-emitting diode (OLED) with low drive voltage and high electroluminescence ${ }^{1}$, organic electroluminescent devices presented a great evolution in structure. In spite of this evolution ITO (indium-tin oxide) continues to be largely used, even with the known constraint of indium diffusion into the organic layers $^{2-5}$.

It was observed that the use of TO (tin oxide) instead of ITO, as transparent electrode, reduces the photoluminescence suppression of PPV converted from a polyelectrolyte precursor $^{6}$, suggesting higher chemical stability of the TO electrode. OLEDs were demonstrated using TO as transparent electrode ${ }^{7-9}$, but the lower work function and the higher electrical resistivity of TO, when compared to ITO, inhibited a wider use of TO in OLEDs. Fluorine-doped tin oxide (FTO) has transparency comparable to that of ITO and can now be produced with sheet resistance similar to that of ITO, being much more stable from the chemical point of view. Intermediate layers like PEDOT:PSS, poly(3,4 ethylenedioxythiophene): poly(styrenesulfonate), or sulfonated polianiline can be used to effectively reduce the potential barrier for positive charge carrier injection ${ }^{10,11}$, so that the low work function of FTO does not more constitute a serious problem limiting FTO use in organic device applications.

In this work we use a complex, but well-established concept of device structure, using FTO as anode, instead of ITO. As the hole injecting layer we used PEDOT-PSS (Baytron $\mathrm{P} \circledast)$ (poly $(3,4$ - ethylenedioxythiophene) : poly(styrenesulfonate)), and OPPVDBC (poly(1,10 decanedioxy - 2 - methoxy - 1,2 - phenylene - 1,2 ethenylene - 3,6 - dimethoxy - 1,4 - phenylene - 1,2 ethenylene - 3-methoxy - 1,4 - phenylene) $)^{12,13}$ was used as hole transport layer, HTL (see Fig. 1). In the organic heterolayer used in this work the recombination zone is expected to be located at the interface between OPPVDBC and $\mathrm{Alq}_{3}$ [tris(8-hydroxyquinoline)aluminum salt], which was used as electron transporting layer (ETL), due to the energetic discontinuity of the relevant energy levels of the layers (HOMO: highest occupied molecular orbital; and LUMO: lowest unoccupied molecular orbital). $\mathrm{Alq}_{3}$, is still an interesting material due to its high electroluminescent efficiency.

\section{Experimental}

Commercial glass substrates coated with FTO (sheet re-

*e-mail: iah@fisica.ufpr.br 
sistance of $15 \Omega / \square$ ) supplied by Flexitec Eletrônica Orgânica Ltda were cut in pieces of $23.0 \times 32.5 \mathrm{~mm}^{2}$, patterned using conventional lithography and etched ${ }^{14}$. After patterning, FTO substrates were cleaned in a 1:4 solution of $\mathrm{H}_{2} \mathrm{O}_{2}: \mathrm{H}_{2} \mathrm{SO}_{4}$ for $1 \mathrm{~h}$ and rinsed in ultra-pure water and isopropanol. No change in surface morphology or transmittance spectra of the FTO was noted due to these cleaning, patterning and etching procedures. Absorbance spectra were measured using an Optometrics spectrophotometer. Film thickness was determined using a Dektak3 surface profiler.

PEDOT-PSS dispersed in $\mathrm{H}_{2} \mathrm{O}$ was treated in ultrasonic bath for $10 \mathrm{~h}$ to dissolve undesired clusters before filtering. PEDOT-PSS was deposited onto glass/FTO by spin-coating at $4.200 \mathrm{rpm}$. After deposition, the sample was dried in a vacuum oven at $313 \mathrm{~K}$ for $4 \mathrm{~h}$. OPPVDBC was dissolved in distilled $\mathrm{CHCl}_{3}$ and deposited onto glass/FTO/PEDOTPSS by spin-coating at $200 \mathrm{rpm}$, resulting in an OPPVDBC film thickness of $\sim 45 \mathrm{~nm}$. $\mathrm{Alq}_{3}, \mathrm{Ca}$ and $\mathrm{Al}$, were evaporated in high vacuum, at $71 \times 10^{-6} \mathrm{~Pa}$, with thickness of 45 $\mathrm{nm}, \sim 200 \mathrm{~nm}$ and $\sim 200 \mathrm{~nm}$, respectively. The fully evaporation sequence was done without vacuum breaking. $\mathrm{Alq}_{3}$ and metal film geometry were controlled through shadow masks geometry during evaporation.

Devices without the $\mathrm{Alq}_{3}$ layer were also made for comparison.

After evaporation, the samples were transported in air to a glove box with dry $\mathrm{N}_{2}$ atmosphere. After $48 \mathrm{~h}$ under $\mathrm{N}_{2}$ flux the samples were encapsulated ${ }^{15,16}$. Electrical and optical measurements were made in the dark. Emission spectra of FTO/PEDOT:PSS $(50 \mathrm{~nm}) / O P P V D B C ~(20 \mathrm{~nm}) /$ $\mathrm{Alq}_{3}(45 \mathrm{~nm}) / \mathrm{Ca} / \mathrm{Al}$ device and of FTO/PEDOT:PSS (50 $\mathrm{nm}) / \mathrm{OPPVDBC}(20 \mathrm{~nm}) / \mathrm{Al} / \mathrm{Ca}$ device were recorded with an OceanOptics CCD spectrophotometer with a $50 \mu \mathrm{m}$ aperture optical fiber and with an Hitachi spectrofluorimeter, respectively.

The Ionization Potential (IP) was estimated using cyclic voltammetry, correcting the oxidation onset at $\mathrm{Ag} / \mathrm{Ag}^{+}$ quasi-reference electrode to vacuum reference level ${ }^{17}$ and the LUMO was estimated summing the energy of the optical gap to the HOMO-energy ${ }^{18}$. The voltammetry was performed in a single compartment electrochemical cell. Gold was used as working electrode and a $0.1 \mathrm{~mol} / 1 \mathrm{LiClO}_{4}$ solution in acetonitrile, as electrolyte.

\section{Results and Discussion}

Taking the oxidation onset of the cyclic voltammogram of OPPVDBC at $\sim 0.53 \mathrm{~V}$ and using the procedure described in $^{17}$, the IP was estimated as $\sim 4.9 \mathrm{eV}$, that summed to the optical gap of $\sim 2.6 \mathrm{eV}^{18}$ leads to an estimated electroaffinity of $\sim 2.3 \mathrm{eV}$. Taking these values and using literature data for the other device layers $\left(\mathrm{Alq}_{3}{ }^{19}, \mathrm{PEDOT}^{20}, \mathrm{FTO}^{21}\right)$, we constructed the device energy scheme, presented in Fig. 2.
The PEDOT layer is introduced because it is able to effectively eliminate the potential barrier between PEDOT and the substrate ${ }^{10}$, in our case, FTO. It strongly collaborates to charge injection in this device, so that positive charge carrier injection in the OPPVDBC layer is not inhibited by the energy barrier at the anode interface. Similarly, the introduction of the $\mathrm{Alq}_{3}$ layer strongly improves the negative charge carrier injection, leading to higher current density through the device, when compared to a device without $\mathrm{Alq}_{3}$ layer, as can be seen in Fig. 3. It was observed that currentvoltage $\mathrm{I}(\mathrm{V})$ characteristics of both devices, FTO/ PEDOT:PSS/OPPVDBC/Alq $/ 3 / \mathrm{Ca} / \mathrm{Al}$ and $\mathrm{FTO} /$ PEDOT:PSS/OPPVDBC/Ca/Al, show negligible rectification.

The device without $\mathrm{Alq}_{3}$ presents lower electroluminescent intensity, leading to a higher noise-level electroluminescent signal (Fig. 4), but could be seen under amber light conditions. More important, however, is that the introduction of the $\mathrm{Alq}_{3}$ layer implies in a significant reduction of the onset voltage for light emission, from $\sim 11 \mathrm{~V}$ to $\sim 6 \mathrm{~V}$. Comparing the spectra of both devices, presented in Fig. 5, we observe a red shift of about $30 \mathrm{~nm}$ in the device with $\mathrm{Alq}_{3}$ layer, indicating that the recombination zone in this device is preponderantly located inside the $\mathrm{Alq}_{3}$ layer ${ }^{22}$. It is important to observe that the

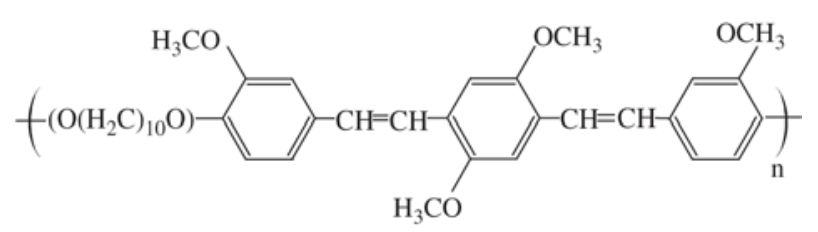

Figure 1. Chemical Structure of OPPVDBC; R. Lessmann et al.

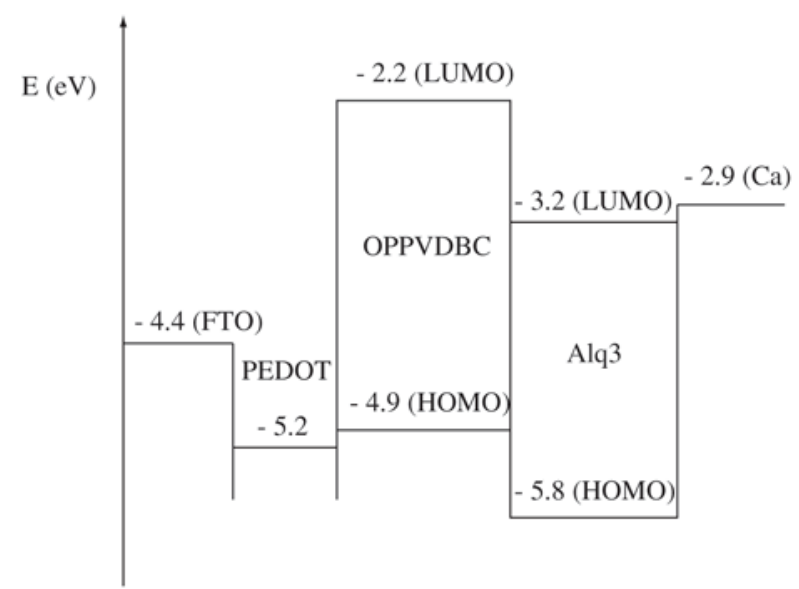

Figure 2. Proposed energy band diagram of the device layers, individually isolated; R. Lessmann et al. 


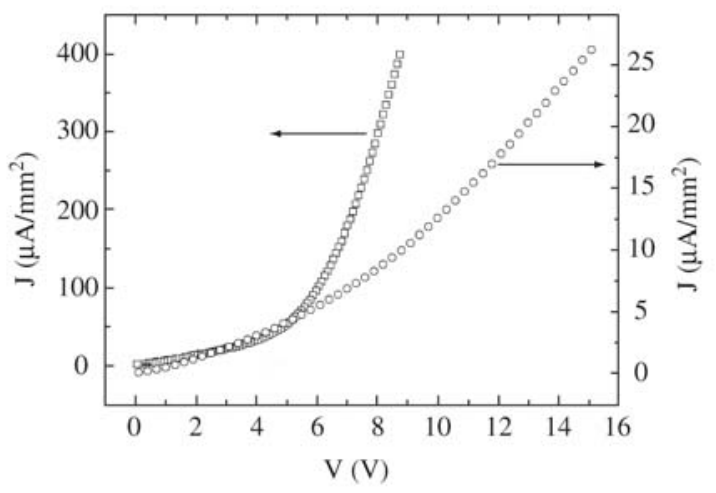

Figure 3. $\mathrm{J} \times \mathrm{V}$ characteristics of a FTO/PEDOT:PSS $(50 \mathrm{~nm}) /$ OPPVDBC $(20 \mathrm{~nm}) / \mathrm{Ca} / \mathrm{Al}$ device (circles) and a FTO/PEDOT:PSS $(50 \mathrm{~nm}) / \mathrm{OPPVDBC}(45 \mathrm{~nm}) / \mathrm{Alq}_{3}(45 \mathrm{~nm}) / \mathrm{Ca} / \mathrm{Al}$ device (squares); R. Lessmann et al.

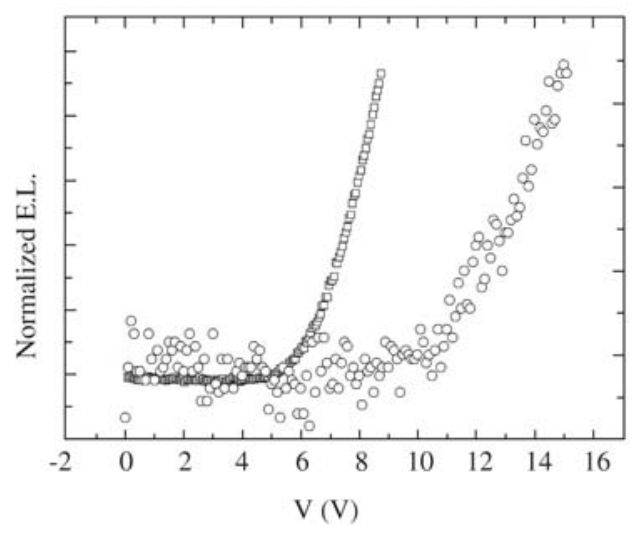

Figure 4. Electroluminescent intensity (EL) as a function of bias voltage (V) of a FTO/PEDOT:PSS (50 nm)/OPPVDBC $(20 \mathrm{~nm}) /$ $\mathrm{Ca} / \mathrm{Al}$ device (circles) and of a FTO/PEDOT:PSS $(50 \mathrm{~nm}) /$ OPPVDBC $(45 \mathrm{~nm}) / \mathrm{Alq}_{3}(45 \mathrm{~nm}) / \mathrm{Ca} / \mathrm{Al}$ device (squares); R. Lessmann et al.

optical gaps of OPPVDBC and of $\mathrm{Alq}_{3}$ are almost the same, permitting, in principle, exciton diffusion across the

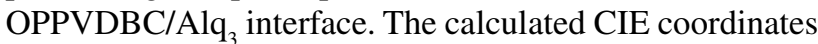
are $\mathrm{x}=0.29=0.58$ for the FTO/PEDOT:PSS $(50 \mathrm{~nm}) /$ OPPVDBC $(45 \mathrm{~nm}) / \mathrm{Alq}_{3}(45 \mathrm{~nm}) / \mathrm{Ca} \mathrm{Al}$ and $\mathrm{x}=0.22$, $\mathrm{y}=0.37$ for the FTO/PEDOT:PSS $(50 \mathrm{~nm}) / \mathrm{OPPVDBC}$ $(20 \mathrm{~nm}) / \mathrm{Ca} / \mathrm{Al}$ device.

In the device with $\mathrm{Alq}_{3}$, the thickness of the HTL (OPPVDBC) and ETL $\left(\mathrm{Alq}_{3}\right)$ is the same. It is interesting to notice that reported mobilities for negative charge carriers in $\mathrm{Alq}_{3}\left(\mu_{\mathrm{e}}^{\mathrm{Alq} 3} \sim 10^{-6} \mathrm{~cm}^{2} / \mathrm{V} . \mathrm{s}\right)^{2}$ and for positive charge carriers in OPPVDBC $\left(\mu_{\mathrm{h}}{ }^{\text {OPPVDBC }} \sim 10^{-9} \mathrm{~cm}^{2} / \mathrm{V} . \mathrm{s}\right)^{18}$ are quite different. In this case one would expect a displacement of

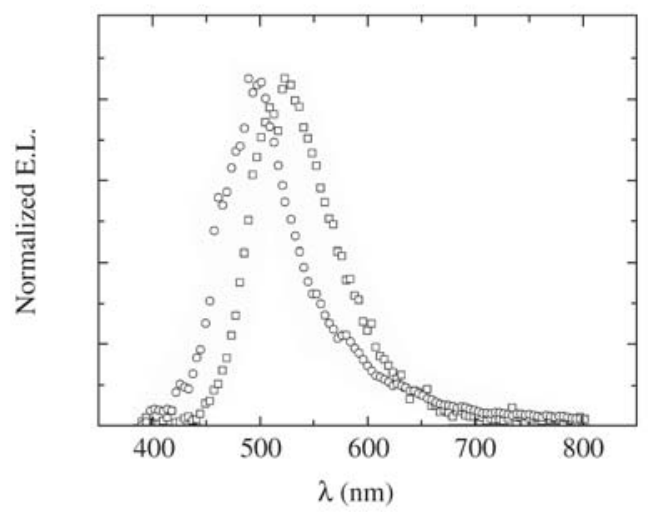

Figure 5. Electroluminescence (EL) spectrum of FTO/PEDOT:PSS $(50 \mathrm{~nm}) / \mathrm{OPPVDBC}(20 \mathrm{~nm}) / \mathrm{Alq}_{3}(45 \mathrm{~nm}) / \mathrm{Ca} / \mathrm{Al}$ device at $8.5 \mathrm{~V}$ (noise filtered). Circles: Electroluminescence spectrum of FTO/ PEDOT:PSS (50 nm)/OPPVDBC $(20 \mathrm{~nm}) / \mathrm{Al} / \mathrm{Ca}$ device at $13 \mathrm{~V}$; R. Lessmann et al.

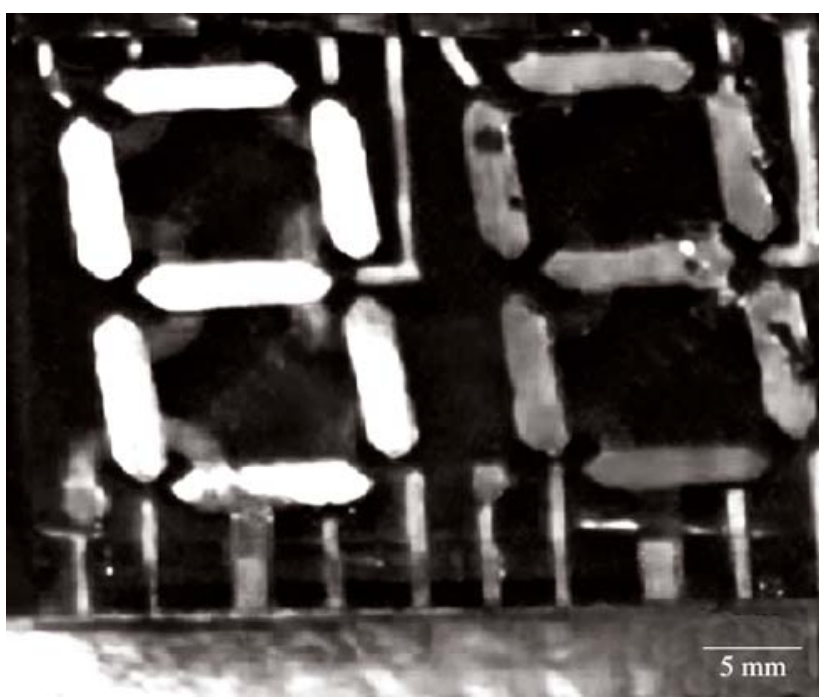

Figure 6. Photograph of two encapsulated 7 segments displays, made of FTO/PEDOT:PSS/OPPVDBC/Alq $3 / \mathrm{Ca} / \mathrm{Al}$, the left one having all segments on, each one driven by a constant current of $7 \mathrm{~mA}$; R. Lessmann et al.

the recombination zone into the OPPVDBC layer, since the electrode interfaces are assumed to present no constraints for charge injection, permitting to assume ohmic injection. Under these conditions it results that radiative recombination is much more favored in $\mathrm{Alq}_{3}$ than in OPPVDBC or that there is a net exciton flow at the OPPVDBC/Alq ${ }_{3}$ to the $\mathrm{Alq}_{3}$ layer due to a difference in exciton binding energy, which would be higher in the $\mathrm{Alq}_{3}$.

We have succeeded in turning on all display segments 
of a seven-segment display. Segments till $16 \mathrm{~mm}^{2}$ were made showing good uniformity (Fig. 6). We made FTO rails wide enough to avoid enhanced resistivity problems. The display was connected to an electronic circuit to count numbers and worked well with the LEDs operating at $\sim 8 \mathrm{~V}$, demonstrating that FTO is suitable for use in LEDs with large active areas and consequently, long conducting paths.

Another point that deserves discussion concerns the work function of FTO and its consequences to device operation. In the device, the PEDOT:PSS layer was used to change the effective energy barrier for charge injection, so that it almost effectively equals the difference $d$ of the chemical potential of the PEDOT:PSS and the anode work function ${ }^{3}$, producing a built-in potential and an electric field in the conjugated materials layers (in our case OPPVDBC and $\mathrm{Alq}_{3}$ ). Considering that thermodynamical equilibrium is established equalizing the electrochemical potential of the electrodes, an additional contribution to the built-in potential appears as a consequence of the difference $\Delta \phi$ in electrodes work functions ${ }^{24}$. These two contributions result that the built-in potential equals $\Delta \phi+\delta$, which is the energy difference between the work function of the cathode and the PEDOT:PSS electrochemical potential, i. e., independent of the anode work function. This means that in principle, in case of devices with intermediate layer like PEDOT:PSS, the anode work function would not be relevant for device operational characteristics. These considerations presume that the PEDOT layer has the capability to compensate the potential difference $\delta$. Since this compensation is done by polarization and charge transfer, it requests a minimum thickness of the PEDOT:PSS layer, otherwise the integration of the electric field throughout the sample thickness can be lower than $\delta$.

Another important aspect that must be stressed is that the substitution of the ITO anode by FTO eliminates the problem of indium diffusion into the electroluminescent polymer layer ${ }^{2-5}$ due to the absence of indium in the anode material composition.

Previous investigations on FTO used in organic LEDs showed that devices prepared onto FTO substrates presented lower turn-on-voltage for current and for light and more light for a given voltage, with one observed drawback, higher leakage currents ${ }^{21}$. In the referred study, however, no intermediate layer was used to improve charge injection from the FTO and device performance was dependent on FTO cleaning procedure, despite the fact that the work function of FTO is not sensible to specific adopted cleaning procedure $^{21}$, like in the case of $\mathrm{TO}^{6}$.

\section{Conclusions}

We have demonstrated that large seven-segment displays can be constructed using low sheet resistance fluorine-doped tin-oxide as anode material. To demonstrate it we constructed FTO/PEDOT:PSS/OPPVDBC/Alq ${ }_{3} / \mathrm{Ca} / \mathrm{Al}$ devices in a geometry useful for low-end electronics.

\section{Acknowledgements}

The authors thanks to CAPES and CNPq for financial support, Winston S. L. Fung for the motivation and Flexitec Eletrônica Orgânica Ltda for providing the FTO substrates. We want to thank Dr. D. Ma who provided OPPVDBC material.

\section{References}

1. Tang, C.; VanSlyke, S. Appl. Phys. Lett. v. 51, p. 913915, 1987.

2. Schlatmann, A.R.; Wilms Floet, D.; Hilberer, A.; Garten, F.; Smulders, P.J.M.; Klapwijk, T.M.; Hadziioannou, G. Appl. Phys. Lett. v. 69, p. 1764-1766, 1996.

3. Chao, C.I.; Ru, K.; Chen, S. A. Appl. Phys. Lett. v. 69, p. 2894-2896, 1996.

4. Herold, M.; Gmeiner, J.; Drummer, C.; Schwoerer, M. J. Mater. Sci. v. 32, p. 5709-5719, 1997.

5. de Jong., M.P.; van Ijzendoorn, L.J.; de Voigt, M.J.A.; Appl. Phys. Lett. v. 77, p. 2255-2257, 2000.

6. Arias, A.C.; Roman, L.S.; Kugler, T.; Toniolo, R.; Meruvia, M.S.; Hümmelgen, I.A. Thin Solid Films v. 371, p. 201-206, 2000.

7. Hümmelgen, I.A.; Yadava, Y.P.; Roman, L.S.; Arias, A.C.; Fernandes, M.R.; Nart, F. C.; Bull. Mater. Sci. v. 19, p. 423-427, 1996.

8. Arias, A.C.; de Lima, J.R.; Hümmelgen, I.A. Adv. Mater. v. 10, p. 392-394, 1998.

9. Vaufrey, D.; Ben Khalifa M.; Besland, M.P.; Sandu, C.; Blanchin, M.G.; Teodorescu, V.; Roger, J.A.; Tardy, J. Synth. Met. v. 127, p. 207-211, 2002.

10. Roman, L.S.; Berggren, M.; Inganäs, O. Appl Phys Lett v. 75, p. 3557-3559, 1999.

11. Roman, L.S.; Mello, R.M.Q.; Cunha, F.; Hümmelgen, I. A.; J. Solid State Electrochem. v. 8, p. 118-121, 2004.

12. Yang, Z.; Hu, B.; Karasz, F. Macromolecules v. 28, p. 6151-6154, 1995.

13. Jiang, X.; Liu, Y.; Song, X.; Zhu, D. Solid State Commun. v. 99, p. 183-187, 1996.

14. Bradshaw, G.; Hughes, A. J. Thin Solid Films v. 33, L5L7, 1976.

15. Toniolo, R.; Hümmelgen, I.A. Brazilian Patent Application, PI0201050-0.

16. Toniolo, R.; Hümmelgen, I.A. Macromol. Mater. Eng., accepted for publication. v. 289, p. 311-314, 2004

17. Micaroni, L.; Nart, F.C.; Hümmelgen, I.A. J. Solid State Electrochem. v. 7, p. 55-59, 2002.

18. Benvenho, A.R.V.; Hümmelgen, I.A. Mater. Res. v. 4, p. 133-136, 2001. 
19. Ohmori, Y.; Fuji, A.; Uchida, M.; Morishima, C.; Yoshino, K. Appl. Phys. Lett. v. 63, p. 1871-1873, 1993.

20. Cacialli, F.; Kim, J. S.; Brown, T. M.; Morgado, J.; Granström, M.; Friend, R. H.; Gigli, G.; Cingolani, R.; Favaretto, L.; Barbarella, G.; Daik, R.; Feast, W.J. Synth. Met. v. 109, p. 7-11, 2000.

21. Anderson, A.; Johansson, N.; Bröms, P.; Yu, N.; Lipo, D.; Salaneck, W. R. Adv. Mater. v. 10, p. 859-863, 1998.
22. Kim, K.; Lee, D.; Jin, J. Synth. Met., v. 114, p. 49-46 2000.

23. Kepler, R.; Beeson, P.; Jacobs, S.; Anderson, R.; Sinclair, M.; Valencia, V. Appl. Phys. Lett. v. 69, p. 3618-3620 1995.

24. Brown, T. M.; Kim, J. S.; Friend, R. H.; Cacialli, F.; Daik, R.; Feast, W. Appl. Phys. Lett. v. 75, p. 1679-1681, 1999. 